\title{
Mechanisms of photoperiod in regulation of rice flowering
}

\author{
Abdulrahman Mahmoud Dogara ${ }^{1, ~ *, ~ S a l i s u ~ M u h a m m a d ~ T a h i r ~}{ }^{1}$, Idris Shehu ${ }^{2}$, Mustapha Abbah ${ }^{3}$, \\ Ummar Shitu ${ }^{4}$, Zainab Idris Ladidi ${ }^{5}$, Aisha Ishaq Jumare ${ }^{1}$
}

${ }^{1}$ Department of Biological science, Kaduna State University, Kaduna State, Nigeria

${ }^{2}$ Department of Microbiology, Kaduna State University, Kaduna State, Nigeria

${ }^{3}$ Department of Micro Biology, Bauchi State University, Gadau, Nigeria

${ }^{4}$ Department of Biology, Isah Kaita College of Education, katsina State, Nigeria

${ }^{5}$ Department of Biochemistry, Bauchi State University, Gadau, Nigeria

\section{Email address:}

abdulrahmanjj@kasu.edu.ng (A. M. Dogara)

\section{To cite this article:}

Abdulrahman Mahmoud Dogara, Salisu Muhammad Tahir, Idris Shehu, Mustapha Abbah, Ummar Shitu, Zainab Idris Ladidi, Aisha Ishaq Jumare. Mechanisms of Photoperiod in Regulation of Rice Flowering. International Journal of Genetics and Genomics.

Vol. 2, No. 1, 2014, pp. 1-5. doi: 10.11648/j.ijgg.20140201.11

\begin{abstract}
A study on the photoperiodic control of flower in rice is advancing and rice has become an example of short day plant. Many genes used in flowering time determination in rice have been identified by many methods. The conclusion from these molecular studies is a remarkable conservation of genes which play an important role in the control of flowering time in rice. The rice photoperiod sensitivity gene $H d 3 a$ was originally detected as a heading date related quantitative trait locus found on chromosome 6 of rice. High resolution linkage mapping of Hd3a was carried using a huge segregating population derived from advanced backcross progeny between a japonica variety, Nipponbare, and an indica variety, Kasalath. Researchers revealed that there are three (3) pathways for the day length regulation of flowering in rice, short day activation pathway and long day suppression pathway lastly the long day activation pathway. Some genes where used in multiple pathways and others are specific to a particular pathway. There regulation also depends on the developmental stages. Rice may be an ideal plant to study the night break effect on flowering. In the near future there would be a need for better understanding of the control of flowering in rice at the genetic level.
\end{abstract}

Keywords: Photoperiod, Heading Date, Short Day Plant, Long Day Plant

\section{Introduction}

Rice is mainly the most important cereal crop which serve as a primary source of food for almost half of the world population[1]. Rice is a tropical legumes plant and mainly source of food which third of the world population depend on it[2]. About three billion people of the world use it as a major or basic food which provide fifty to eighty percent of the calories needed[3]. It grown in almost one hundred and fourty eight million hectares annually or on eleven percent of the world planted land [2]. It is a member of order graminaecea of grass family consisting of two (2) cultivers mainly: Oryza stiva which originate from Asia and is found all over the world, the other one Oryza gleberrima mostly found in only one region of the world; specifically west africa and is grown in small propotion. Oryza as a rice cultiver has been in existance for about one hundred and thirty million years ago, and is evenly distributed all over the world apart from the Antarctica[2]. Rice is the cereal plant that is mainly consume by human, Wheat cover a significant propotion of land but majority of it is utilized as animal feed [2]. Rice is very important source of our food. Rice is grown in about one hundred and fifty-four million hectares annually or in eleven percent of the worlds cultivated areas, in two hundred world rice production was approximated to be six hundred million tones[1]. It production increased at the rate of two point three to two point five percent during nineteen-seventy's and nineteeneighty's however during the nineteen ninety's the growth rate decline yearly by one ponit five percent up to present decade[4]. The number of rice consumption is increasing at the rate of one ponit five percent annually where as its production at present increases only at the rate of one percent annually[4]. According to the United Nation (UN) 
estimation, the world population will increase from six point seven billion at present to about eight billion by twenty twenty-five, therefore its production must increase from four hundred and fourty million tones at present to four hundred and seventy five million tones by twenty twenty[4]. Food and Agricultural Organization (FAO) estimated that by twenty-fifty the world rice requirement will be five hundred and twenty-four million tones which required annual increase of two million tones from the present level of production[4]. Flowering date act as the most considerable aspect of agricultural trait of rice. Rice farmers all over the world have been advancing rice collections whose flowering time is most applicable for the place where they are planted. The formation of a flower is important for succcessful sexual reproduction, therefore the actual timing of it has adaptive value, especially in non self fertile species where flowering has to be synchronize among them [5]. Even in self fertile species, the induction of flowering is regulated by enviromental and endogenous factors such as day length, temperature and hormonal constituents[5]. Regulation of flowering time has been investigated about one hundred years ago. Early studies revealed that plant flowering is controlled by factors that include day length (i.e photoperiod)[5]. Photoperiod is a time limit of a plant to get expose to light. Photoperiodism is one of the most important characteristic of plants because it helps to provide the plants with the suitable signals that are necessary for flowering. Plants in general consists of three sensing photoperiods classes; short and long day which play a vital role in promoting flowering by sensing the short and long day photoperiods respectively. lastly, the neural day flowering plants whose photoperiods have no effects on it [6]. Mostly all cultivars of rice are short day plants, and mature in a shorter time under a short photoperiod (about 10 hours) compared to long photoperiod (14 hours). However, the photoperiod sensitivity varies greatly among cultivars[5]. Advancement in the establishment of rice genomic sequence and the possible production of transgenic rice makes it an interesting model organism for molecular studies in monocots [7]. To meet with challenge of producing more rice from the existing land resources, therefore rice vareties with a high yield potential are required, to achieve this there is need to know how photoperiod regulate flowering in rice which is very vital to a successful development of high yields potential cultivers. In this review we summarize the various molecular and genetic approach that are currently adopted through understanding of photoperiod to increase the potential yield of rice.

\section{Pathways Regulating the Photoperiodic Rice Flowering}

In rice, flowering is determined by short day and withhold by long day condition. short day inhibiting pathway and long day withholding pathway are present in rice while there are so many rice that flowering is determine during long day although it takes longer time than short day and therefore there is a third pathway in rice which is long day inhibiting pathway[6]. Pathways for long day suppression and long day inhibition can be differentiated by advancing stage of the plant. Long day withholding sustain from undeveloped stage to the developmental stage[10]. Then the long day inbition pathway becomes activated at later stage and it is independent of the long day suppression pathway, therefore in long day, both the activating pathway and the suppression pathway function simultaneously in the late developmental stage[17]. As it will be described, the $O s G I-$ $H d 1-H d 3 a$ pathway are the main pathway for the regulation of flower in rice and it forms both short day inhibiting pathway and long day suppression pathway depending on the regulation of $H d 3 a$ by $H d 1$ [10]. Behavior of these pathways is also developmentally controlled. The short day inhibition pathway becomes activated at undeveloped stage while the long day suppression pathway functions throughout the entire the growth stage[8]. To best knowledge of the present authors, the molecular situation of the last pathway i.e the long day inhibiting pathway, has not been clearly understood, and it is going to be important to know constituents of this pathway[9].

\section{Major Pathways Responsible for the Flowering Control in Rice}

Three(3) genes which serve as a major genetic pathway in the photoperiodic control of flower in rice have been identified as: OsGI, an ortholog of Arabidopsis GI, Hdl, an ortholog of Arabidopsis CO, and Hd3a,an ortholog of Arabidopsis FT are shown to form the major pathway for the photoperiodic control of flower in rice[10]. OsGI was isolated as a gene whose mRNA was withhold in the se5 mutant which is insensitive to the photoperiod by many array method [11]. OsGI has high similarity with $G I$ and contains a nuclear localization signal. $H d l$ was identified by map-based cloning after the isolation by quantitative trait locus (QTL) analysis which is closely associated to Arabidopsis CO, which play the major role in the photoperiodic regulation of flower. Two motifs, zinc finger motif and CCT motif, are highly conserved in $H d 1$ and $\mathrm{CO}[10] . H d 3 a$ was similarly isolated by map-based cloning after the identification by QTL analysis[11].Hd3a and FT are highly conserved and both function as activators of flowering under inductive conditions[11].Heading date are found on the short arm of chromosome 6 which involved in the photoperiodic response and promoting flowering in short day conditions are identified through a map-based strategy which show a high level of similarity with $F T$ gene that promote flowering during long day condition[10]. Transgenic analysis also show that the introduction of $H 3 a$ gene result in early flowering in SDPs and LDPs conditions, moreover $H d 3 a$ mRNA is up-regulated in the short-day 
conditions that induce flowering in rice this that $H d 3 a$ plays an important role in the production of flowering in rice[10]. Overexpression of $H d 3 a$ and FT lead to early heading in rice and early flowering in Arabidopsis, the fact revealed that $H d 3 a$ promotes the floral transition as in FT sequence and function similarity show that $H d 3 a$ is an ortholog of $F T[11]$. It also revealed that several copies of $F T$ like genes found in the rice genome: such as RFT1 located closed to $H d 3 a$ which its presumed amino acid sequence with ninety-one percent similarity with $H d 3 a$ [11]. Advancement in the establishment of rice genomic sequence and the possible production of transgenic rice makes it an interesting model organism for molecular studies in monocots [7]. Heading date 3 gene ( $\mathrm{Hd} 3 \mathrm{a}$ ), which is found on the chromosome six (6) play an important role in short day flowering plant by promoting photoperiodic response [10]. $H d 3 a$ together with $h d l$ are positive regulator under short day condition and the transgenic analysis rice plants shows that over expressing or suppressing of OsGI gene activates hd1 in short day as well as in long day rice but however, $O s G I$, together with $H d 1$ suppresses the expression of $H d 3 a$ gene in long day condition thereby serving as negative regulators for the flowering [10]. These shows that $O s G I-H d 1-H d 3 a$ is the major genetic pathway for the regulation of photoperiod in flowering rice plants and these is also conserved in Arabidopsis which is a long day plant[12]. Hd1 is only activated in the dark period which give room for the expression of $H d 3 a$ gene [13]. The dual function of $H d 1$ for the regulation of $H d 3 a$ gene is dependent on the photoperiod.

\section{Minor Genes Involved in the Photoperiod Pathway in Rice}

Heading date 6 was identified by QTL analysis and revealed to encode the a-subunit of protein kinase CK2. It causes delayed flowering in long day plant[17]. The Arabidopsis CK2 was shown to be involved in the circadian rhythm; therefore it is also conserved in rice and Arabidopsis[17]. Genes which have been isolated as key regulators of the photoperiodic regulation of flowering are well conserved between rice and Arabidopsis[14].Therefore, one challenge arise: are there any rice specific genes involved in control of flowering? Ehdl was isolated by map based cloning and shown to contain a B-type response regulator[15]. Ehdl acts to promote flowering in short day by activating expression of $H d 3 a$ and its homolog's. Its interesting feature is that it activates downstream genes independently of $\mathrm{Hdl}$, suggesting the presence of a branched pathway for promotion of flowering in short day. Although there are other genes genetically identified by QTL analysis, they have not been molecularly identified[15]. There is a good possibility that genes which are unique to rice flowering will be discovered in the near future

\section{Night Break Effect in Rice}

A short exposure to light in the middle of the night promotes flowering in short day plants. The phenomenon is called 'night break' (NB) and has been used previously and presently by researchers as a tool to study the photoperiodic regulation of flowering for many years. However, at the molecular level not much is known about this phenomenon. In Oryza sativa $10 \mathrm{~min}$ of light exposure in the middle of a 14-h night caused a delay in flowering[14]. A single NB strongly suppressed the mRNA of $H d 3 a$, a homolog of Arabidopsis thaliana FLOWERING LOCUS T (FT), whereas the mRNAs of $O s G I$ and $H d 1$ were not affected. The NB effect on Hd3a mRNA was maximal in the middle of the 14-h night[15]. The phyB mutation abolished the NB effect on flowering and $H d 3 a$ mRNA, indicating that the NB effect was mediated by phytochrome B. Because expression of the other $F T$-like genes was very low and not appreciably affected by NB, our results strongly suggest that the suppression of $H d 3 a$ mRNA is the principal cause of the NB effect on flowering in rice[15].

\section{Genetic Time of Rice Flowering}

The genetics of flowering is quantitative in nature. To know the determinants of heading date in rice, several groups have used quantitative trait loci (QTL) analyses [10]. Exploiting phenotypic differences and DNA polymorphisms within cultivated rice varieties and wild relatives of rice, researchers revealed that the loci are responsible for regulating heading date; determine their relative contribution to phenotypic variance, which establish genetic relationships between loci[16]. Adopting different segregating populations obtained by crossing a Nipponbare and Kasalath M. Yano's group identified most of the QTLs controlling heading date in rice, and pioneered the genetics of photoperiodic flowering [17]. $H d 1$ to $H d 5$ are the dominant loci regulating photoperiodic flowering responses which act as single Mendelian factors in a segregating F2 population[18]. The Nipponbare alleles of $H d 1$ and $H d 2$ were shown to be short day flowering promoters with the largest effects on phenotype, acting independently of one another [17]. $\mathrm{Hd} 3 a$ genetically interacts with $H d 1$ and $H d 2$ to inhibit flowering under short day conditions[19]. The Kasalath alleles of Hd4 and $H d 5$ delayed flowering under long day conditions [16]. The genetic effect of $\mathrm{Hd} 4$ identified to be additive to that of $H d 1$ and $H d 2$, whereas an epistatic relationship was detected among $H d 5$ and $H d 1$ [20]. With the exception of heading date 2 ( $H d 2$ ) these QTLs have been fine mapped and the underlying genes cloned[21]. Backcross progenies derived from the same original cross allowed initial identification and cloning of other QTLs, often with minor effects, for many of these, the underlying genes still need to be identified[22]. Researchers revealed that by analyzing rice populations mutagenized thorough transgenes, transposons or $\gamma$-radiation, researchers 
identify several genes implicated in flowering time control or day length perception, and position them into regulatory networks [17].Phenotypic and functional analyses have suggested that photoperiodic flowering in rice is control by a genetic cascade that have similarities with that described for Arabidopsis, both in the components involved and in their mode of action. It has also become clear that a parallel pathway unique to rice can control flowering on its own and both pathways eventually converge on the control of a group of genes encoding florigenic proteins [16].

\section{Conclusion}

Researches on the photoperiodic control of flowering in rice have advanced and rice has become a model of shortday plant. Photoperiod regulates much developmental response in animals, plants and even fungi. The response to photoperiod has evolved because daylength is a reliable indicator of the time of the day, enabling developmental events to be scheduled to coincide with particular environmental conditions. Much progress has been made towards understanding the molecular mechanisms involved in the response to photoperiod in plants. These mechanisms include the detection of the light signal in the leaves, the entrainment of circadian rhythms, and the production of a mobile signal which is transmitted throughout the plant. Many genes involved in flowering time determination in rice have been identified by various methods. One striking conclusion from these molecular studies is a remarkable conservation of genes which play important roles in the regulation of flowering time in rice. Scientist revealed that there are three pathways for the day length control of flowering in rice: Short day activation pathway, long day suppression pathway and the long day activation pathway. Some genes are used in multiple pathways and others are pathway specific. They are also differentially regulated depending on developmental stages. Rice may be an ideal plant to study the night break effect on flowering. In the near future we hope better understand the regulation of flowering rice at the molecular level.

\section{References}

[1] Kole, C., Screening rice for drought resistance at the reproductive phase. Field Crops Research, 1994. 39(2): p. 99-110.

[2] Khush, G.S., Origin, dispersal, cultivation and variation of rice, in Oryza: From Molecule to Plant. 1997, Springer. p. 25-34.

[3] Sohrabi, M., et al., Genetic Diversity of Upland Rice Germplasm in Malaysia Based on Quantitative Traits. The Scientific World Journal, 2012. 2012.

[4] Jeon, J.-S., et al., Genetic and molecular insights into the enhancement of rice yield potential. Journal of Plant Biology, 2011. 54(1): p. 1-9.
[5] Srikanth, A. and M. Schmid, Regulation of flowering time: all roads lead to Rome. Cellular and Molecular Life Sciences, 2011. 68(12): p. 2013-2037.

[6] Komiya, R., et al., Hd3a and RFT1 are essential for flowering in rice. Development, 2008. 135(4): p. 767774 .

[7] Shimamoto, K. and S. Yokoi, The Photoperiodic Control of Flowering in Rice, a Short-Day Plant, in Light Sensing in Plants. 2005, Springer. p. 339-346.

[8] Searle, I. and G. Coupland, Induction of flowering by seasonal changes in photoperiod. The EMBO Journal, 2004. 23(6): p. 1217-1222.

[9] Second, G., Origin of the genic diversity of cultivated rice (Oryza spp.): study of the polymorphism scored at 40 isozyme loci. Jpn J genet, 1982. 57: p. 25-57.

[10] Yano, M., et al., Genetic control of flowering time in rice, a short-day plant. Plant Physiology, 2001. 127(4): p. 14251429 .

[11] Kojima, S., et al., Hd3a, a rice ortholog of the Arabidopsis FT gene, promotes transition to flowering downstream of Hd1 under short-day conditions. Plant and Cell Physiology, 2002. 43(10): p. 1096-1105.

[12] Izawa, T., Adaptation of flowering-time by natural and artificial selection in Arabidopsis and rice. Journal of experimental botany, 2007. 58(12): p. 3091-3097.

[13] Izawa, T., et al., Phytochrome mediates the external light signal to repress FT orthologs in photoperiodic flowering of rice. Genes \& development, 2002. 16(15): p. 2006-2020.

[14] Yano, M., et al., Hd1, a major photoperiod sensitivity quantitative trait locus in rice, is closely related to the Arabidopsis flowering time gene CONSTANS. The Plant Cell Online, 2000. 12(12): p. 2473-2483.

[15] Ishikawa, R., et al., Suppression of the floral activator Hd3a is the principal cause of the night break effect in rice. The Plant Cell Online, 2005. 17(12): p. 3326-3336.

[16] Brambilla, V. and F. Fornara, Molecular Control of Flowering in Response to Day Length in Rice. Journal of integrative plant biology, 2013.

[17] Yano, M., et al., Identification of quantitative trait loci controlling heading date in rice using a high-density linkage map. Theoretical and Applied Genetics, 1997. 95(7): p. 1025-1032.

[18] Yamamoto, T., et al., Identification of heading date quantitative trait locus Hd6 and characterization of its epistatic interactions with $\mathrm{Hd} 2$ in rice using advanced backcross progeny. Genetics, 2000. 154(2): p. 885-891.

[19] Gowda, M., et al., Advances in rice breeding, genetics and genomics. Molecular Breeding, 2003. 11(4): p. 337-352.

[20] Wang, J.-D., et al., Ectopic expression of OsMADS45 activates the upstream genes Hd3a and RFT1 at an early development stage causing early flowering in rice. Botanical Studies, 2013. 54(1): p. 12.

[21] SONG, Y.-1. and W.-j. LUAN, Molecular Regulatory Network of Flowering by Photoperiod and Temperature in Rice. Rice Science, 2012. 19(3): p. 169-176. 
[22] Matsubara, K., et al., Natural variation in Hd17, a homolog of Arabidopsis ELF3 that is involved in rice photoperiodic flowering. Plant and Cell Physiology, 2012. 53(4): p. 709716. 